\title{
DESCUENTO DE FLUJO DE FONDOS E INFLACIÓN PARA LA VALORACIÓN DE EMPRESAS EN DOS MONEDAS*
}

\author{
Recibido: 15 de noviembre de 2016 • Aprobado: 28 de agosto de 2017 \\ DOI: 10.22395/seec.v20n44a9
}

\section{Gastón Silverio Milanesi**}

\section{RESUMEN}

El objetivo del trabajo consiste en presentar un marco basado en las teorías de la paridad sobre el modelo de descuento de flujos para obtener valoraciones en dos monedas. Se presentan soluciones técnicas para la proyección de magnitudes financieras y costo de capital, expresados en términos reales y nominales. Para estudiar e ilustrar su funcionamiento se emplea el método de análisis de caso. Los resultados obtenidos indican que en equilibrio de mercado, el valor actual de los flujos de fondos descontados en dos monedas, obtenidos a partir de magnitudes financieras proyectadas y expresadas en diferente poder adquisitivo, gozan de consistencia si todas las variables que expliquen el valor de la firma (flujos de fondos, costo del capital promedio ponderado y valor de continuidad) son calculadas considerando el impacto de la inflación. Para ello es menester que la tasa de cambio esperada, de contado y futura, las tasas de interés y la inflación esperada, local y extranjera, sean obtenidas aplicando la teoría de la paridad.

\section{PALABRAS CLAVE}

Valoración de empresas; inflación; descuento de flujos de fondos; teoría de la paridad; Argentina

\section{CLASIFICACIÓN JEL}

G31, F31, M21

\section{CONTENIDO}

Introducción; 1. Desarrollo del modelo de valoración; 2. Metodología: análisis de caso de valoración en dos monedas para empresa en marcha; 3. Conclusiones; Bibliografía; Anexos.

\footnotetext{
* El presente artículo es producto del proyecto de investigación titulado "Reformulación de los modelos de valuación de empresas en Argentina: problemas de especificación, efecto inflación, sesgos y heurísticas" 24/C/040 evaluado, acreditado y financiado por la Secretaría General de Ciencia y Tecnología de la Universidad Nacional del Sur, Argentina, ejecutado en el período 2014-2016.

* Contador público nacional, magíster en Administración y doctor en Ciencias de la Administración Universidad Nacional del Sur, Argentina. Profesor titular exclusivo e investigador, Departamento Ciencias de la Administración, Universidad Nacional del Sur, Bahía Blanca, Buenos Aires, Argentina. Dirección: Calle San Andrés s/n Campus Universitario Palihue, 8000 Bahía Blanca, Argentina. Correo electrónico: milanesi@uns.edu.ar
} 


\section{DISCOUNT OF CASH FLOWS AND INFLATION FOR THE VALUATION OF COMPANIES IN TWO CURRENCIES}

\section{ABSTRACT}

The objective of this article is to present a framework based on parity theories on the model of discounts of flows in order to obtain valuations in two currencies. Technical solutions are presented for the projection of financial magnitudes and capital cost, expressed in real and nominal terms. In order to study and illustrate its operation, the case analysis method is employed. Results obtained indicate that during a market balance, the current value of cash flows discounted in two currencies, obtained from financial magnitudes projected and expressed in different purchase power, are consistent if all variables that explain the company's value (cash flows, weighted average capital cost, and continuity value) are calculated by taking the impact of inflation into consideration. For this purpose, expected, cash, and future exchange rate, interest rates, and local and foreign expected inflation should be obtained by applying the parity theory.

\section{KEY WORDS}

Valuation of companies; inflation; cash flow discount; parity theory; Argentina.

\section{JEL CLASSIFICATION}

G31, F31, M21

\section{CONTENT}

Introduction; 1. Development of the valuation model. 2. Methodology: Analysis of valuation case in two currencies for operating company. 3. Conclusions. Bibliography. Annexes.

\section{DESCONTO DE FLUXO DE FUNDOS E INFLAÇÃO PARA A VALORIZAÇÃO DE EMPRESAS EM DUAS MOEDAS}

\section{RESUMO}

O objetivo deste trabalho consiste em apresentar um referencial baseado nas teorias da paridade sobre o modelo de desconto de fluxos para obter valorizações em duas moedas. Apresentam-se soluções técnicas para a projeção de magnitudes financeiras e custo de capital, expressos em termos reais e nominais. Para estudar e ilustrar seu funcionamento, emprega-se o método de análise de caso. Os resultados obtidos indicam que, em equilíbrio de mercado, o valor atual dos fluxos de fundos descontados em duas moedas, obtidos a partir de magnitudes financeiras projetadas e expressas em diferente poder aquisitivo, gozam de consistência se todas as variáveis que explicam o valor da firma (fluxos de fundos, custo do capital médio ponderado e valor de continuidade) forem calculadas considerando o impacto da inflação. Para isso, é necessário que a taxa de câmbio esperada, a vista e futura, bem como as taxas de juros e a inflação esperada, doméstica e estrangeira sejam obtidas aplicando a teoria da paridade.

\section{PALAVRAS-CHAVE}

Valorização de empresas; inflação; desconto de fluxos de fundos; teoria da paridade, Argentina

\section{CLASSIFICAÇÃO JEL}

$$
\text { G31, F31, M21 }
$$

\section{CONTEÚDO}

Introdução; 1. Desenvolvimento do modelo de valorização; 2. Metodologia: análise de caso de valorização em duas moedas para empresa em marcha; 3. Conclusões; Bibliografia; Anexos. 


\section{INTRODUCCIÓN}

En condiciones de mercados perfectos, eficientes y completos se deben verificar un conjunto de relaciones de equilibrio, tomando como base la ley del precio único. En efecto, las teorías de paridad de las tasas de cambio, tasas de interés y poder de compra representan el nexo de equilibrio para magnitudes financieras expresadas en dos monedas diferentes. Por lo tanto, la valoración de empresas en marcha mediante el modelo de descuento de flujos de fondos, en contextos emergentes e inflacionarios y con valores expresados en diferentes monedas, requiere de un marco conceptual apoyado en las teorías de la paridad. De esta manera, se asegura la consistencia entre el valor actual obtenido a partir de magnitudes financieras expresadas en moneda local o extranjera, de poder adquisitivo futuro (nominal o de cierre) o poder adquisitivo a fecha de valoración (real o inicial).

Existen excelentes tratamientos parciales sobre el tema en cuestión como los de Modigliani y Cohn (1984); Copeland, Koller y Murrin (2000); Titman y Grinblatt (2002); Vélez-Pareja (2006); Damodaran (2006); Emery y Finnerty (2007); Bradley y Gregg (2008); Pratt y Grabowski (2008); Tahn y Vélez-Pareja (2011) y López (2014b), entre otros.

El objetivo de este trabajo consiste en desarrollar una metodología para valorar empresas, mediante el modelo de descuento de flujos de fondos, empleando variables en moneda de cierre e inicial como dos monedas diferentes. Su punto de apoyo son las relaciones fundamentales contenidas en las finanzas internacionales: teorías de paridad de tasas de interés, poder de compra, expectativas de las tasas de cambio y efecto Fisher. Estas atraviesan el modelo en todas sus variables: proyección de beneficios, tasa de costo de capital y valor de continuidad. Metodológicamente se emplea el estudio de casos, con el fin de ilustrar y testear el funcionamiento del modelo. Los resultados obtenidos, a partir de variables expresadas en términos nominales y reales, como en dos monedas, son coherentes con las relaciones de paridad que sostienen al modelo.

La estructura del trabajo es la siguiente: primero son expuestas las teorías de paridad y estimación de curvas de rendimientos. Se desarrolla el marco metodológico para estimar flujos de fondos proyectados, costo del capital y valor terminal, con el fin de aplicar de manera consistente el modelo de descuento de flujos de fondos, con magnitudes nominales y reales en diferentes monedas. Para ilustrar el funcionamiento del modelo propuesto se aplica la metodología del estudio de casos. Finalmente se presentan las principales conclusiones. 


\section{DESARROLLO DEL MODELO DE VALORACIÓN}

\subsection{Las teorías de la paridad y el efecto Fisher}

En esta sección se presentan en forma resumida las teorías de paridad en tasas de interés, poder de compra, efectos Fisher y curvas de rendimiento, las cuales aportan las bases del modelo objeto de estudio:

a) Paridad en la tasa de interés (TPI): plantea la relación entre las tasas de interés local $\left(r_{t, d, n}\right)$ y extranjeras $\left(r_{t, e, n}\right)$ nominales, con el fin de explicar la relación entre la tasa de cambio de contado, también conocida como spot (S), y la tasa de cambio futura nominal $\left(\mathrm{F}_{t, n}\right)$. La siguiente ecuación ilustra la relación explicada:

$$
\frac{\mathrm{F}_{\mathrm{t}, \mathrm{n}}}{\mathrm{S}}=\frac{\left(1+\mathrm{r}_{\mathrm{t}, \mathrm{d}, \mathrm{n}}\right)}{\left(1+\mathrm{r}_{\mathrm{t}, \mathrm{n}, \mathrm{n}}\right)}
$$

b) Paridad en el poder de compra (PPC): bajo la ley de precio único, un bien comercializado en dos mercados diferentes debe venderse al mismo precio en ambos mercados, en términos de poder adquisitivo. La tasa de cambio es el común denominador que explica las diferencias nominales. Cuando se verifica esta paridad, las tasas de inflación ajustan el valor de las monedas, manteniendo la paridad en el poder de compra como indican Emery y Finnerty (2007). La paridad del poder de compra se expresa formalmente como el cociente entre las tasas de inflación esperadas $(\pi)$, tanto doméstica $\left(\pi_{t, d}\right)$ como extranjera $\left(\pi_{t, e}\right)$, las cuales explican el diferencial entre $(S)$ y la tasa de cambio spot esperada $E\left[S_{t, n}\right]$ :

$$
\frac{\mathrm{E}\left[\mathrm{S}_{t, n}\right]}{\mathrm{S}}=\frac{\left(1+\pi_{t, d}\right)}{\left(1+\pi_{t, \mathrm{x}}\right)}
$$

De la ecuación anterior, en el caso de mercados emergentes, es posible deducir la tasa de inflación para un período,

$$
\pi_{t, d}=\frac{\mathrm{E}\left[\mathrm{S}_{t, n}\right]}{\mathrm{S}} \times\left(1+\pi_{t, x}\right)-1
$$

c) Teoría de expectativas de la tasa de cambio esperada, de contado y futura: cuando el mercado se encuentra en equilibrio se verifica el principio de convergencia entre $F_{t, n}$ y $E\left[S_{t, n}\right]$ tal que:

$$
\mathrm{E}\left[\mathrm{S}_{t, n}\right]=\mathrm{F}_{t, n}=\mathrm{S} \times \frac{\left(1+r_{t, d, n}\right)}{\left(1+r_{t, x, n}\right)}
$$


d) Efecto Fisher: las tasas de interés nominal observadas en el mercado reflejan las expectativas inflacionarias de los agentes. Esto se conoce como efecto Fisher'. Estas ideas formalizan la distinción entre tasas de interés real y nominal, y se conoce como ecuación de Fisher, según Argandoña (2013). En ese orden de ideas, una tasa nominal surge a partir de la tasa real $\left(r_{t, r}\right)$, según la siguiente expresión:

$$
r_{t, d, n}=\left(r_{t, r}+\pi_{t, d}\right)+\left[r_{t, r} \times \pi_{t, d}\right]
$$

El efecto Fisher indica que la diferencia en el valor de las tasas nominales correspondientes a dos economías se explica por las distintas tasas de inflación. Por lo tanto, la tasa en términos reales queda expresada así:

$$
\frac{\left(1+r_{t, d, n}\right)}{\left(1+\pi_{t, d}\right)}=\frac{\left(1+r_{t, x, n}\right)}{\left(1+\pi_{t, x}\right)}=1+r_{t, r}
$$

Para una economía determinada, la ecuación de arbitraje de Fisher entre tasas nominales y reales, es la siguiente²:

$$
\left(1+\pi_{t, d}\right)\left(1+r_{t, r}\right)=\left(1+r_{t, d, n}\right)
$$

Despejando en función de la tasa nominal, la expresión alternativa la ecuación 5 es la siguiente,

$$
r_{t, d, n}=\left(1+\pi_{t, d}\right)\left(1+r_{t, r}\right)-1
$$

Cuando la tasa de inflación no es significativa, a menudo se desprecia el segundo término de la ecuación y se expresa como $r_{t, d, n}=r_{t, d}+\pi_{t, d}$ y $r_{t, d}=r_{t, d, n-} \pi_{t, d}$ respectivamente.

\subsection{La curva de rendimientos proyectada en mercados emergentes}

Desde el punto de vista instrumental, estimar la TPI y PPC requiere de insumos como son las tasas de intereses proyectadas, ya que se supone que el diferencial entre tasas de interés esperadas en moneda local y extranjera captura la depreciación (apreciación) de la moneda doméstica frente a la divisa. Para ello se debe calcular

\footnotetext{
1 Irving Fisher (1965) planteó el hecho que las tasas nominales de interés reflejan la expectativa colectiva inflacionaria, y que dicha tasa compensa a los agentes de los efectos negativos de la inflación sobre el rendimiento real de sus inversiones.

2 Cuando existen posibilidades de arbitraje, estas son explotadas por los agentes hasta llegar a una situación de equilibrio en las tasas reales y diferencias en las nominales explicada por la tasa de inflación.
} 
una curva teórica de rendimientos o estructura temporal de tasas de interés (ETTI) utilizando títulos en moneda doméstica y extranjera emitidos. Las técnicas que frecuentemente son utilizadas se conocen como bootstrapping y ajuste logarítmico.

a) Bootstrapping: propuesta por Fabozzi y Fabozzi (1996), para su construcción se emplean bonos cupón cero, con horizontes de corto plazo disponibles en el mercado. Para horizontes de mediano y largo plazo se resuelve, en orden ascendente, utilizando tasas spot estimadas. Estas son calculadas por despeje respecto del último pago, a partir del uso de bonos tipo bullets.

b) Ajuste logarítmico: consiste en derivar la curva de rendimientos de bonos, mediante regresión logarítmica entre la duration modificada y la TIR (tasa interna de retorno de los bonos) en moneda doméstica y extranjera ${ }^{3}$.

\subsection{Moneda en poder adquisitivo de cada período proyectado (de cierre) 0 en poder adquisitivo a fecha de valoración (inicial). Efectos traslación y absorción}

Las magnitudes financieras proyectadas pueden expresarse en moneda con poder adquisitivo de cada período proyectado, conocida como de cierre o nominal, y en moneda con poder adquisitivo a fecha de valoración, conocida como de inicio, inicial o en términos reales. A continuación, son explicadas las técnicas para estimar magnitudes financieras en monedas de diferente poder adquisitivo:

a) Magnitudes en moneda de poder adquisitivo de cada período proyectado (cierre): las magnitudes financieras en moneda de cierre son obtenidas mediante un coeficiente. Este se construye a partir de un número índice, con base 1 en el inicio $(t=0)$;

$$
i d_{t}=i d_{t-1} \times\left(1+\pi_{t, i}\right)
$$

Donde id representa el índice (del período ty $t$-1 período anterior) y $\pi_{t, i}$ evolución en el nivel de precios para $(t)$ y activo $(i)$. El coeficiente $\left(n x_{t}\right)$, surge con la siguiente expresión,

$$
n x_{t}=i d_{t} / i d_{t-1}
$$

De la expresión anterior, se obtiene la cifra expresada en moneda de cierre $\left(v_{t, n}\right)$,

$$
v_{t, n}=v_{t-1, n} \times n x_{t}
$$

Se emplea la curva logarítmica debido a que esta es la que mejor se ajusta a la relación duración modificada-TIR, ya que al aumentar la duración el crecimiento de la TIR es rápido al principio para luego suavizarse frente a mayores duraciones (López, 2014a). 
b) Magnitudes en moneda de poder adquisitivo a fecha de valoración (inicial): para calcular magnitudes financieras en moneda de poder adquisitivo inicial se requiere la construcción del índice y su respectivo coeficiente. Primero se construye el índice como la relación entre evolución del precio del flujo $\pi_{t, i}$ y el índice general de precios (inflación general) ${ }^{4}\left(\pi_{t, g}\right)$ :

$$
\triangle \pi_{t, i}=\left(\pi_{t, i} / \pi_{t, g}\right)-1
$$

Seguidamente, se calcula el coeficiente $\left(r x_{t}\right)$ para deflactar valores nominales de la siguiente forma:

$$
r \chi_{t}=\Delta \pi_{t, i} / \triangle \pi_{t-1, i}
$$

Y así la magnitud en moneda inicial $\left(v_{t, r}\right)$ será:

$$
v_{t, r}=v_{t+1, r} \times r x_{t}
$$

De las relaciones entre valores nominales y reales nacen los efectos absorción (ecuación 15) y traslación (ecuación 16);

$$
\begin{aligned}
& v_{t, r}=v_{t, n} / r x_{t} \\
& v_{t, n}=v_{t, r} \times n x_{t}
\end{aligned}
$$

\subsection{Magnitudes monetarias, resultados proyectados y el flujo de fondos en moneda de cierre e inicial}

Aplicar el modelo de descuento de flujo de fondos requiere definir las siguientes variables: flujos de fondos del horizonte explícito de proyección, costo del capital y el valor terminal ${ }^{5}$. La presenten sección se concentrará en los aspectos a tener en cuenta para proyectar resultados y flujos de fondos nominales y reales.

a) Ingresos y costos en moneda de cierre: el primer ejercicio prospectivo implica proyectar unidades esperadas de producción $\left(q_{p, t}\right)$ y ventas $\left(q_{v, t}\right)$ por período.

\footnotetext{
$4 \quad$ Nótese que la ecuación anterior mide cambio en precios relativos pronosticados, a diferencia de la ecuación 9, donde se analiza la evolución nominal de un precio.

5 El modelo de descuento de flujos de fondos es tratado con profundidad por un importante cuerpo de trabajos sobre valoración de empresas, entre ellos se puede citar Copeland, Koller y Murrin, (2000); Pratt y Grabowski (2008) entre otros. Siguiendo a Ruback (2002); Damodaran (2006); Booth (2007) y Fernández (2015) existen tres métodos consistentes para trabajar el costo del capital: a) Costo Capital Promedio Ponderado (ccpp, wacc), b) Flujos de fondos a capital, c) Valor Presente Ajustado (APV). En el presente trabajo se adoptará la técnica del ccpp.
} 
Seguidamente se está en condiciones de estimar los niveles de inventarios ${ }^{6}$, ya que estos integrarán el capital de trabajo no monetario de la firma. Luego se procede a valorar los ítems indicados incorporando la inflación esperada. Para ello se proyecta precio unitario de venta $\left(\mathrm{PVU}_{t, n}\right)$, costo variable unitario $\left(\mathrm{CVU}_{t, n}\right)$ y el costo fijo total unitario $\left(\mathrm{CFU}_{t, n}\right)$. Finalmente, se estima el costo total $\left(\mathrm{CTU}_{t, n}\right)$. Los costos e ingresos en pesos, para el período $t$, surgen del producto entre precio en $t-1$ y el coeficiente (ecuación10),

$$
\begin{gathered}
\mathrm{PVU}_{t, n}=\mathrm{PVU}_{t-1, n} \times n x_{t} \\
\mathrm{CVU}_{t, n}=\mathrm{CVU}_{t-1, n} \times n x_{t} \\
\mathrm{CFU}_{t, n}=\mathrm{CFT}_{t, n} / q_{p, t}
\end{gathered}
$$

Multiplicando precios (ecuaciones 17,18 y 19) y cantidades proyectadas, se obtienen los flujos totales de ingresos, costos variables, fijos y totales de producción. Respecto de las existencias, primero se proyectan las unidades, acorde con niveles de ventas y producción proyectadas:

$$
\mathrm{EF}_{q, t}=\mathrm{EI}_{q, t}+q_{p, t}-q_{v, n}
$$

En la expresión anterior, $q_{p, t}$ y $q_{v, n}$ representan las unidades producidas y vendidas; $\mathrm{EF}_{q, t}$ y $\mathrm{EI}_{q, t}$ existencias finales e iniciales. La valoración de los inventarios del producto tiene las existencias finales y el costo total unitario. El costo de artículos vendidos se obtiene del producto entre costo total unitario y cantidades vendidas,

$$
\mathrm{CAVt}, n=\mathrm{CTU}_{t, n} \times q_{v, t} .
$$

b) Impuesto a la ganancia: algunas legislaciones tributarias no reconocen el impacto de la inflación en la determinación del impuesto a las ganancias; por lo tanto, el costo de artículos vendidos se computa a valores históricos. Por tal motivo, la proyección de la base gravable y de los resultados se debe realizar independientemente. Consecuentemente $\mathrm{EI}_{q, t}$ se computan a su precio histórico y tanto $q_{p, t}$ como $\mathrm{EF}_{q, t}$ por el costo total unitario del producto $\mathrm{CTU}_{t, n}$ corriente, siendo el costo histórico $\mathrm{CAV}_{t, \mathrm{H}}=\left(\mathrm{EI}_{q, t} \times \mathrm{CTU}_{t-1, n}\right)+\left(q_{p, t} \times \mathrm{CTU}_{t, n}\right)-\left(\mathrm{EF}_{q, t} \times \mathrm{CTU}_{t, n}\right)$. La base gravable es:

$$
\mathrm{BI}_{t}=\mathrm{IT}_{t, n}-\mathrm{CAV}_{t, \mathrm{H}}-\mathrm{G}_{t, \mathrm{H}}-\mathrm{A}_{t, \mathrm{H}}
$$

No es el objetivo del presente trabajo indagar sobre las técnicas prospectivas utilizadas en la proyección de estados financieros. Sin perjuicio de ello, la metodología de mayor difusión está dada por el uso de la técnica de escenarios. Ver, entre otros, a Miller y Waller (2003); Wright, Cairns y Goodwin (2009) y Esandi, Milanesi y Pesce (2012). 
Donde $\mathrm{IT}_{t, n}$ son los ingresos del período, $\mathrm{CAV}_{t, \mathrm{H}}$ costo de artículos vendidos histórico, $\mathrm{G}_{t, \mathrm{H}}$ otros gastos, $\mathrm{A}_{t, \mathrm{H}}$ amortización histórica y BI base gravable. Seguidamente, la determinación del impuesto $\left(\mathrm{ID}_{t}\right)$ surge aplicando la alícuota (T) sobre la base gravable, siendo $\mathrm{ID}_{t}=\mathrm{B}_{i t} \times \mathrm{T}$. En el caso de que la legislación tributaria disponga el ingreso de anticipos de impuestos, el impuesto a pagar $\left(\mathrm{IP}_{t}\right)$ es la diferencia entre el impuesto determinado y anticipos ingresados en el período anterior $\left(\mathrm{AP}_{t-1}\right) ; \mathrm{IP}_{t}=\mathrm{ID}_{t}-\mathrm{AP}_{t-1}$. Es importante tener en cuenta esto, ya que se genera un crédito monetario integrante del capital de trabajo, conforme será analizado en el punto d).

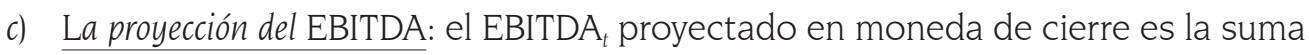
algebraica de $\mathrm{IT}_{t, n}-\mathrm{CAV}_{t, n}-\mathrm{G}_{t, n}$. De allí se obtiene:

$$
\mathrm{RO}_{t}=\mathrm{EBITDA}_{t, n}-\mathrm{A}_{t, n}-\mathrm{ID}_{t, n}
$$

Donde $\mathrm{RO}_{t}$ es el resultado operativo después de impuestos y $\mathrm{A}_{t, n}$ representa la amortización del período en moneda de cierre, menos el valor del impuesto a las ganancias proyectado.

d) Capital de trabajo rubros monetarios y no monetarios: los elementos que integran el capital de trabajo proyectado deben estar separados según su exposición a la inflación en monetarios y no monetarios. En el caso de las partidas monetarias, la inflación produce un resultado negativo del activo (efectivo y cuentas a pagar sin indexación en moneda doméstica) y positivo producto del pasivo monetario (deudas sin indexación en moneda doméstica). Por lo tanto, la variación total correspondiente a la inversión incremental proyectada en capital de trabajo se descompone en

$$
\Delta \mathrm{CT}=\triangle \mathrm{CTM}+\triangle \mathrm{CTNM}
$$

Donde $\Delta C T$ representa el incremento total en capital de trabajo, integrado por la variación de los componentes monetarios $\triangle$ CTM y no monetarios $\triangle$ CTNM. Respecto de los últimos, cabe destacar dos tratamientos para calcular el resultado por exposición a la inflación. Una primera alternativa consiste en calcular $\Delta$ CTM como la diferencia entre la magnitud inicial de cada partida expresada en moneda de poder adquisitivo del ejercicio anterior $\mathrm{CTM}_{(t-1), m i}$ y la magnitud final expresada en moneda de poder adquisitivo de cierre (ejercicio presupuestado) $\mathrm{CTM}_{(t), m c^{7}}{ }^{7}$. En este caso la variación total contiene el incremento real (aumento físico) y la variación nominal por exposición a la inflación (resultado proyectado por exposición a la inflación neto, $\left.\left(\mathrm{REI}_{t}\right)\right)$ :

\footnotetext{
Positivo, en el caso que los pasivos monetarios sean superiores a los activos monetarios, y negativo, en el caso inverso.
} 


$$
\triangle \mathrm{CTM}_{(\text {total }), n}=\mathrm{CTM}_{(t), m c}-\mathrm{CTM}_{(t-1), m i}
$$

Otra alternativa consiste en expresar las magnitudes iniciales, en moneda de poder adquisitivo de cierre; $\Delta \mathrm{CTM}_{(\text {real }), n}=\mathrm{CTM}_{(t), m c}-\mathrm{CTM}_{(t-1), m c}$. La variación obtenida representa la efectiva inversión incremental o liberación de fondos. En este caso el resultado proyectado por exposición a la inflación se explicita como un componente más en el flujo de fondos proyectados en moneda de cierre (ecuación 24), siendo $\Delta \mathrm{CTM}_{\text {(total) }, n}-\Delta \mathrm{CTM}_{\text {(realt, }, \mathrm{r}}=\mathrm{REI}_{t}$. Para rubros no monetarios (inventarios), la variación real (inversión-desinversión) se obtiene comparando las magnitudes iniciales y finales expresadas en moneda de igual poder adquisitivo $\Delta \mathrm{CTM}_{(\text {real) }, n}=\mathrm{CTNM}_{(t), m c}-\mathrm{CTNM}_{(t-1), m c}$.

e) Flujos de fondos libres en moneda de cierre $\left(\mathrm{FFL}_{t, n}\right)$ : surge de sumar las magnitudes monetarias vistas en el apartado anterior agregando las inversiones incrementales en activos fijos operativos, $\Delta \mathrm{AF}_{t, n}$ :

$$
\mathrm{FFL}_{t, n}=\mathrm{EBITDA}_{t, n}-\mathrm{ID}_{t, n}-\triangle \mathrm{CTM}_{(\text {total) } t, n}-\triangle \mathrm{CTNM}_{t, n}-\triangle \mathrm{AF}_{t, n}
$$

En la ecuación 25, la variación sobre el capital de trabajo monetario $\left(\Delta \mathrm{CTM}_{\text {(total)t, }}\right.$ engloba a la variación real y al efecto generado debido al resultado por exposición a la inflación. Si la variación del capital de trabajo refleja solamente el incremento real, entonces el resultado por exposición a la inflación $\left(\mathrm{REI}_{t}\right)$ debe incorporarse como un ítem del flujo de fondos libres en la expresión 25.

f) Flujos de fondos libres en moneda inicial: el flujo de fondos libres en moneda de cierre (ecuación 25) es deflactado (ecuación15) con el fin de obtener el flujo de fondos en moneda de inicio (real) $\left(\mathrm{FFL}_{t, r}\right)$ :

$$
\mathrm{FFL}_{t, r}=\frac{\mathrm{FFL}_{t, n}}{r x_{t, a}}
$$

En el caso de estimar cada uno de los elementos del flujo de fondos en términos reales, la expresión queda planteada de la siguiente manera:

$$
\mathrm{FFL}_{t, r}=\mathrm{EBITDA}_{t, r}-\mathrm{ID}_{t, r}-\triangle \mathrm{CTM}_{(\text {real }), r}-\mathrm{REI}_{t}-\triangle \mathrm{CTNM}_{t, r}-\triangle \mathrm{AF}_{t, r}
$$

Para ello es menester utilizar las ecuaciones 12, 13 y 14 para la creación del índice, el coeficiente y la posterior transformación de magnitudes en moneda de cierre a moneda inicial. El impuesto a las ganancias se calcula en términos reales; de similar manera, la inversión incremental real en capital de trabajo, y el resultado del período, por exposición a la inflación. 
g) Valor actual de los flujos en moneda local (doméstico) $\left(\mathrm{VA}_{d}\right)$ : es menester que exista consistencia entre el poder adquisitivo en que se expresan flujos y tasa. Los flujos proyectados en moneda de cierre deben actualizarse al costo del capital nominal $\left(k o_{n, t}\right)$ :

$$
\mathrm{VA}_{d}=\sum_{t=1}^{n} \frac{\mathrm{FFL}_{t, n}}{\left(1+k o_{n, t}\right)^{t}}
$$

En el caso de trabajar con flujos proyectados en moneda inicial (reales) deben actualizarse al costo del capital real $\left(\left(k o_{r, t}\right)\right.$ :

$$
\mathrm{VA}_{d}=\sum_{t=1}^{n} \frac{\mathrm{FFL}_{t, r}}{\left(1+k o_{r, t}\right)^{t}}
$$

Las ecuaciones 28 y 29 conducen al mismo resultado.

\subsection{Costo del capital e inflación}

En este apartado será analizado el tratamiento del costo del capital promedio ponderado (CCPP). En términos nominales su expresión se representa mediante la siguiente ecuación:

$$
k o_{n, t}=\mathrm{W}_{e} \times k e_{n, t}+\mathrm{W}_{d} \times k i_{n, t} \times(1-\mathrm{T})
$$

Donde $\mathrm{W}_{e} y \mathrm{~W}_{d}$ representan ponderaciones de las fuentes de financiamiento capital propio y deuda, mientras que $k e_{n, t}$ y $k i_{n, t}$ representan el costo del capital propio y de la deuda expresado, y T, la alícuota del impuesto a las ganancias. Aplicando la ecuación de Fisher, el costo del capital propio y ajeno en términos nominales se descompone en $k e_{n, t}=k e_{r, t}+\pi_{t, d}+\left(k e_{r, t} \times \pi_{t, d}\right)$ y $k i_{n, t}=k i_{r, t}+\pi_{t, d}+\left(k i_{r, t} \times \pi_{t, d}\right)$.

La magnitud CCPP en términos reales se puede estimar de forma directa o indirecta. De forma directa implica deflactar la ecuación 30 como plantean Bradley y Gregg (2008) y Tahn y Vélez-Pareja (2011).

$$
k o_{r, t}=\frac{k o_{n, t}-\pi_{t, d}}{1+\pi_{t, d}}
$$

Para estimarla en forma indirecta, se procede a calcular las tasas en términos reales, de cada componente del CCPP (ecuación 30). Uno de los errores de especificación más frecuente resulta del incorrecto tratamiento del ahorro fiscal sobre el costo del capital ajeno. El procedimiento incorrecto implica tomar la tasa nominal 
de la deuda antes de impuestos y transformarla en real, para luego incorporar el efecto del ahorro fiscal (Modigliani y Cohn, 1984), conforme se expone en la siguiente expresión kidp ${ }_{r, t}=\frac{k i_{n, t}-\pi_{t, d}}{1+\pi_{t d}}(1-\mathrm{T})$. El punto de partida siempre debe ser el costo nominal de la deuda después de impuestos $\left(k i_{n, t}(1-T)\right.$, para luego estimar la tasa real, $k i d p_{r, t}=\frac{k i_{n, t}(1-\mathrm{T})-\pi_{t, d}}{1+\pi_{t, d}}$. La expresión del CCPP (deflactada) planteada por Tahn y Vélez-Pareja (2011) es:

$$
k o_{r, t}=\mathrm{W}_{e} \times\left(\frac{k e_{n, t}-\pi_{t, d}}{1+\pi_{t, d}}\right)+\mathrm{W}_{d} \times\left(\frac{k i_{n, t}(1-\mathrm{T})-\pi_{t, d}}{1+\pi_{t, d}}\right)
$$

\subsection{Valor de continuidad ${ }^{8}$ e inflación}

En la práctica profesional es usual emplear el concepto de valor de continuidad o terminal para resumir el valor actual de los flujos de fondos esperados a perpetuidad, generados luego de lo que se conoce como horizonte explícito de proyección. La expresión utilizada frecuentemente surge del modelo de crecimiento constante, propuesta por Gordon (1962), expresión descompuesta en conductores de valor por Rappaport (1998); Copeland, Koller y Murrin (2000); Brealey, Myers y Allen (2006) y Pratt y Grabowski (2008), entre otros. El valor terminal (VT) se expresa como:

$$
\mathrm{VT}=\frac{\mathrm{FFL}_{n, t}}{k o_{n, t}-\mathrm{G}}
$$

Donde $G$ es la tasa de crecimiento nominal. Con $G=0$, sin inversiones incrementales o con inversiones marginales de $\mathrm{VAN}=0$, este es:

$$
\mathrm{VT}=\frac{\mathrm{FFL}_{n, t}}{k o_{n, t}}
$$

La expresión anterior se conoce como Zero Nominal Growth Model, (ZNG) criticada por los errores que se incurren en la indebida especificación del modelo. Estos consisten en las incorrectas definiciones de tasa de crecimiento y del incremento en los flujos nominales, producto del revalúo del valor nominal del capital invertido. Siguiendo a Bradley y Gregg (2008) las alternativas propuestas para subsanar dichos inconvenientes son:

\footnotetext{
$8 \mathrm{Al}$ valor de continuidad se lo conoce como valor terminal. Este representa el valor actual de la corriente de flujos de fondos que se supone estable, calculados a partir de la fecha de finalización del horizonte explícito de proyección e infinito (Copeland, Koller y Murrin, 2000).
} 
a) Zero Real Growth Model (ZRG): aplicando la paridad de Fisher, el rendimiento operativo nominal o $\mathrm{ROA}\left(\mathrm{R}_{t}\right)$ sobre los activos de la firma $\left(\mathrm{CI}_{t-1}\right)$ es:

$$
\mathrm{R}_{t}=r+\pi_{t, d}+r \times \pi_{t, d}
$$

En donde $r$ representa la tasa de rendimiento real. Los conductores de valor que explican el crecimiento de la firma son la tasa de reinversión y los flujos de fondos libres. La primera surge del cociente entre la inversión marginal y los flujos de fondos operativos ( $t r=\Delta \mathrm{CI}_{t} / \mathrm{FFO}_{t, n}$ ), siendo el flujo de fondos operativos la suma de ingresos y costos antes de inversiones incrementales. El flujo de fondos libres se puede expresar como el producto entre el flujo de fondos libres y el complemento de la tasa de reinversión:

$$
\mathrm{FFL}_{n, t}=\mathrm{FFO}_{t, n} \times(1-t r)
$$

Consecuentemente, la correcta especificación de la tasa de crecimiento nominal es:

$$
\mathrm{G}=\operatorname{tr} \times \mathrm{R}_{t}+(1-\operatorname{tr}) \times \pi_{t, d}
$$

La ecuación precedente explica el crecimiento en términos nominales mediante dos componentes: a) tasa de crecimiento en los flujos de fondos libres nominales generados por las nuevas inversiones $\left(r \times \mathrm{R}_{t}\right)$; b) aumento o revalúo del valor nominal correspondiente a los activos fijos de la firma, a partir del efecto de la inflación, (1-tr) $\times \pi_{t, d}$. Por lo general, en el segundo término se ignora, cuando se plantea la tasa utilizando la clásica expresión: $G=\operatorname{tr} \times R_{t}$. Entonces, el efecto de la inflación en la tasa de crecimiento de la firma dependerá de la tasa de reinversión ( $t r$ ). Si esta es 0 , entonces $G=\pi_{t, d}$ y todo el crecimiento es atribuible a al incremento en los valores de mercado del stock de capital. Si la tasa de reinversión es $>0$, entonces el aumento en el valor de los activos es acompañado por un incremento en los flujos de fondos de nuevas inversiones. En términos reales la ecuación 37 queda reducida, $g=\operatorname{tr} \times r$.

Sustituyendo en la ecuación 33 por el flujo (ecuación 36) y tasa de crecimiento (ecuación 37) se tiene:

$$
\mathrm{VT}=\frac{\mathrm{FFO}_{n, t} \times(1-t r)}{k o_{n, t}-\left(t r \times \mathrm{R}+(1-t r) \times \pi_{n, t}\right)}
$$

Si la tasa de reinversión es cero la ecuación queda reducida a

$$
\mathrm{VT}=\frac{\mathrm{FFL}_{n, t}}{k o_{n, t}-\pi_{n, t}}
$$


Debido a que $G=\pi_{n, t}$, esta debe capturar en términos nominales el efecto inflación sobre capital invertido, en términos reales $g=0, t r=0$. Supone que los flujos de fondos reales son constantes a perpetuidad, pero el flujo de fondos en términos nominales no es constante. Crece a razón de la tasa de inflación sobre el stock de capital. Se considera que el modelo es neutral a la inflación, puesto que sus variables expresadas en términos reales arriban al mismo resultado que en términos nominales ${ }^{9}$, conforme se expondrá en la siguiente sección. Las ecuaciones 39 y 40 presentan la congruencia del enfoque en términos nominales y reales $\mathrm{FFL}_{n, t}=\mathrm{FFL}_{r, t}(1+\pi), k o_{n, t}=k o_{r, t}(1+\pi)$.

$$
\mathrm{VT}=\frac{\mathrm{FFL}_{r, t}(1+\pi)}{k o_{r, t}(1+\pi)}=\frac{\mathrm{FFL}_{r, t}}{k o_{r, t}}
$$

b) Zero net present value investment (ZNPI): en este caso se supone que las inversiones proyectadas son de $\mathrm{VAN}=0$, donde el rendimiento de la inversión iguala a la tasa de costo del capital $\left(\mathrm{R}=k o_{n, t}\right)$. En la ecuación 33, reemplazando numerador y denominador por las ecuaciones 36 y 37 , se tiene:

$$
\mathrm{VT}=\frac{\mathrm{FFO}_{n, t} \times(1-t r)}{k o_{n, t}-\left(t r \times \mathrm{R}+(1-t r) \times \pi_{t, d)}\right.}=\frac{\mathrm{FFO}_{n, t} \times(1-t r)}{k o_{n, t}(1-t r)-(1-t r) \times \pi_{t, d}}
$$

Operando el denominador se llega a la misma conclusión que para la ecuación 39.

$$
\mathrm{VT}=\frac{\mathrm{FFL}_{n, t}}{k o_{n, t}-\pi_{t, d}}
$$

\subsection{Valor actual en dos monedas nominales y reales}

Las TPI y PPC (ecuaciones 1 a 5 ) brindan el marco consistente para valorar en moneda doméstica (d) y extranjera $(x)$ de cierre e inicio. Para ello existen dos caminos:

a) Conversión de flujos nominales en moneda local a flujos nominales en moneda extranjera: se parte de los flujos de fondos proyectados estimados en la ecuación 25. Primero se debe proyectar la evolución de la tasa de cambio futura. Para ello se utiliza la curva de rendimientos estimada para cada período (ecuación 1) como proponen Hull (2005) y López (2014b). Seguidamente los flujos nominales son convertidos a moneda extranjera empleando la tasa de cambio futura $\left(F_{t, n}\right)$, para obtener los flujos nominales en moneda extranjera (FFL $\left.x_{t, n}\right)$.

\footnotetext{
Esto es así ya que si en la ecuación (39) el denominador $k o_{n, t}-\pi_{n, t}$ se sustituye por $\left|k_{r, t}+\pi_{t, d}+k o_{r, t} \times \pi_{t, d}\right|$ $-\pi t, d$, operando se reduce a $k_{r, t}\left(1+\pi_{t, d}\right)$. Seguidamente los coeficientes de capitalización a inflación esperada $\left(1+\pi_{t, d}\right)$ en el numerador y denominador se simplifican.
} 


$$
\mathrm{FFL} x_{t, n}=\frac{\mathrm{FFL}_{t, n}}{\mathrm{~F}_{t, n}}
$$

Los flujos nominales se actualizan al CCPP, ambas magnitudes expresadas en moneda extranjera. El CCPP extranjero se obtiene del nominal doméstico aplicando la siguiente expresión, $\left(1+k o_{n, t}\right)=\left(1+k o x_{n, t}\right) \times \frac{\left(1+\pi_{t, d}\right)}{\left(1+\pi_{t, x}\right)}$. Operando sobre las tasas de inflación se obtiene:

$$
\left(1+k o x_{n, t}\right)=\left(1+k o_{n, t}\right) \times \frac{\left(1+\pi_{t, d}\right)^{-1}}{\left(1+\pi_{t, x}\right)}
$$

Donde $k o x_{n, t}$ es el ccpp nominal en moneda extranjera. Aplicando las ecuaciones anteriores, se obtiene el valor actual en moneda extranjera $\left(\mathrm{VA}_{x}\right)$ :

$$
\mathrm{VA}_{x}=\sum_{t=1}^{n} \frac{\mathrm{FFL} x_{t, n}}{\left(1+k o x_{n, t}\right)^{t}}
$$

b) Conversión de flujos reales en moneda local flujos reales en moneda extranjera: en este caso los insumos son los flujos reales domésticos (ecuación 27). Primero, se estima la tasa de cambio futura real, es decir, ajustada por la inflación extranjera y doméstica. Segundo, se estima la tasa de cambio futura nominal (ecuación 1). Y finalmente, se ajusta por la inflación extranjera y se deflacta por la doméstica:

$$
\mathrm{F}_{t, r}=\mathrm{F}_{t, n} \times \frac{\left(1+\pi_{t, x}\right)}{\left(1+\pi_{t, d}\right)}
$$

El flujo en moneda extranjera real (FFL $\left.\chi_{t, r}\right)$ es:

$$
\mathrm{FFL} x_{t, r}=\frac{\mathrm{FFL}_{t, r}}{\mathrm{~F}_{t, r}}
$$

El CCPP en moneda extranjera real $\left(k_{0} x_{r, t}\right)$ se obtiene deflactando el CCPP nominal en moneda extranjera (ecuación 7):

$$
k o x_{r, t}=\frac{k o x_{n, t}-\pi_{t, x}}{\left(1+\pi_{t, x}\right)}
$$

Actualizando los flujos en moneda inicial con el CCPP real se llega al mismo valor que en términos nominales conforme será ilustrado en la siguiente sección los valores de las ecuaciones 45 y 49 son consistentes. 


$$
\mathrm{VA}_{\chi}=\sum_{t=1}^{n} \frac{\mathrm{FFLx}_{t, r}}{\left(1+k o x_{r, t}\right)^{t}}
$$

Todo el procedimiento asegura que el valor actual en moneda doméstica $\left(\mathrm{VA}_{d}\right)$ dividido por la tasa de cambio spot sea igual al valor actual en moneda extranjera. Esto significa que al ser los valores de las ecuaciones 45 y 49 iguales, entonces se sostiene la siguiente igualdad:

$$
\frac{\mathrm{VA}_{d}}{\mathrm{~S}}=\sum_{t=1}^{n} \frac{\mathrm{FFLx}_{t, n}}{\left(1+k 0 x_{n, t}\right)^{t}}=\sum_{t=1}^{n} \frac{\mathrm{FFLx}_{t, r}}{\left(1+k 0 x_{r, t}\right)^{t}}
$$

Recíprocamente, se debe verificar que el valor actual en moneda extranjera multiplicado por la tasa de cambio spot iguale el valor actual en moneda doméstica.

$$
\sum_{t=1}^{n} \frac{\mathrm{FFL}_{t, n}}{\left(1+k o_{n, t}\right)^{t}}=\sum_{t=1}^{n} \frac{\mathrm{FFL}_{t, r}}{\left(1+k o_{r, t}\right)^{t}}=\mathrm{VA}_{x} \times \mathrm{S}
$$

\section{METODOLOGÍA: ANÁLISIS DE CASO DE VALORACIÓN EN DOS MONEDAS PARA EMPRESA EN MARCHA}

Se utiliza la metodología del estudio de $\operatorname{casos}^{10}$ porque no se buscan soluciones generalizables estadísticamente. El modelo presentado en el apartado anterior describe el conjunto de variables que determinan el valor de una empresa como son las tasas de interés, inflación, flujos y costo de capital, las cuales vamos a aplicar a una firma en marcha como unidad de análisis. La unidad de análisis es un emprendimiento de mediano-pequeño tamaño (PyME) en el marco del sistema económico argentino (mercado emergente). Su actividad principal es comercial; se dedica a la comercialización en el sector mayorista de artículos derivados de los plásticos industriales, específicamente bolsas de uso industriales destinadas al almacenamiento o empaque. En el trabajo, se pretende determinar su valor intrínseco a través del modelo de descuento de flujo de fondos, trabajando las variables en términos nominales y reales, y expresando valores en moneda local y extranjera. La primera moneda es el peso argentino, y la segunda, el dólar estadounidense. Seguidamente se presenta el modelo con cinco apartados: estimación de las variables macro (a); proyección de ingresos, costos, resultados y flujos (b); estimación del CCPP (c); determinación del valor terminal (d); flujos y valor actual en moneda extranjera (e) y cálculo de consistencia entre el valor actual en moneda doméstica y extranjera.

\footnotetext{
10 El estudio de casos como metodología de investigación no debe confundirse con el análisis o estudio de casos como herramienta pedagógica, la cual busca analizar un aspecto concreto relacionado con las organizaciones para fomentar el debate o discusión, según Castro (2010).
} 
a) Proyección de las variables macro: tasas de interés, inflación y tasa de cambio: suponiendo que las relaciones de Fisher se sostienen, la primera tarea consiste en proyectar las tasas esperadas de interés doméstica y extranjera. Argentina se caracteriza por tener un mercado de capitales emergentes; en consecuencia, no se encuentran disponibles bonos cupón cero, ni STRIPS o americanos que abarquen el horizonte de proyección de 6 años. Siendo descartada la técnica de bootstrapping, se opta por la estimación de la curva logarítmica de rendimientos, empleando datos correspondientes a duración modificada y TIR de bonos soberanos en moneda doméstica y dólares estadounidenses publicados por el Instituto Argentino de Mercados de Capitales (IAMC); estos datos se presentan en las tablas A1 y A2 del anexo ${ }^{11}$. Los datos de las TIR se regresan contra el tiempo con el fin de obtener las ecuaciones de pronóstico; estas son: i) TIR pesos: $-0,064 \ln (x)+0,2283$; ii) TIR dólares: 00,0104 $\ln (x)+0,0402 ;-212,6 \ln (x)+1618,18$. La técnica de ajuste logarítmico también se empleó para proyectar la inflación local (ecuación 3). Primero se calculó la curva por ajuste logarítmico correspondiente a la inflación extranjera, utilizando datos correspondientes a la inflación promedio anual de Estados Unidos desde el año 1999 hasta el año 2016 (agosto) ${ }^{12}$. En la siguiente tabla se presentan los resultados de tasas esperadas, inflación doméstica esperada a partir de la inflación extranjera (ecuación 3) y efecto Fisher internacional (ecuación 6). La tasa de cambio esperado (ecuaciónl) surge de tomar el spot a fecha de valoración y utilizar las tasas proyectadas para obtener el futuro en el primer período. Así, sucesivamente, para el cálculo del resto de las series.

b) Proyección de ingresos, costos, resultados operativos y flujos de fondos: con los datos de la tabla 1 se procede a construir los coeficientes para expresar los valores en moneda de cierre (ecuaciones 9, 10 y 11) e inicial (ecuaciones 12, 13 y 14). Los valores resultantes de la proyección de unidades vendidas, producidas, precios, ingresos totales, costos variables, fijos y existencias finales e iniciales de bienes de cambio en moneda de cierre e histórica se presenta en la tabla A3 del anexo (ecuaciones 17 a 20). La determinación de la base gravable correspondiente al impuesto a las ganancias proyectado (ecuación 21), los resultados operativos después de impuestos proyectados (ecuación 22) y la inversión incremental

11 Los datos correspondientes a TIR y DM de bonos soberanos y domésticos se obtienen del sitio web del Instituto Argentino de Mercados de Capitales (IAMC). El rendimiento de los bonos indexados por el coeficiente de estabilización de referencia (CER) fue ajustado por una inflación proyectada del $17 \%$ para el primer año, conforme los datos oficiales del relevamiento de expectativas de mercado desarrollados por el Banco Central de la República Argentina.

12 Obtenida del sitio web US Inflation Calculator, con los cuales se obtuvo la siguiente curva y $=-212,6 \ln (\mathrm{x})$ $+1618,8$. 
en capital de trabajo (ecuaciones 23 y 24) son presentados en la tabla A4 del anexo, expresados en miles. Seguidamente se presentan los flujos proyectados nominales (ecuación 25). De ellos se obtiene su expresión en términos reales (ecuaciones 16 y 26). Finalmente, se obtiene el flujo de fondos proyectado en moneda corriente e inicial conforme se presenta en la tabla 2.

Tabla 1. Proyección de variables

\begin{tabular}{|c|c|c|c|c|c|c|}
\hline Horizontes & $\mathrm{E}\left(i_{d}\right)$ & $\mathrm{E}\left(i_{x}\right)$ & $\mathrm{F}_{t, n}$ & $\prod_{t, e}$ & $\prod_{t, d}$ & $r_{t, r}$ \\
\hline $31 / 12 / 2016$ & - & - & $\$ 15,55$ & - & - & - \\
\hline 2017 & $22,83 \%$ & $4,02 \%$ & $\$ 18,36$ & $1,05 \%$ & $19,32 \%$ & $2,94 \%$ \\
\hline 2018 & $18,39 \%$ & $4,74 \%$ & $\$ 20,76$ & $0,94 \%$ & $14,10 \%$ & $3,76 \%$ \\
\hline 2019 & $15,80 \%$ & $5,16 \%$ & $\$ 22,85$ & $0,84 \%$ & $11,04 \%$ & $4,29 \%$ \\
\hline 2020 & $13,96 \%$ & $5,46 \%$ & $\$ 24,70$ & $0,73 \%$ & $8,85 \%$ & $4,69 \%$ \\
\hline 2021 & $12,53 \%$ & $5,69 \%$ & $\$ 26,29$ & $0,63 \%$ & $7,14 \%$ & $5,03 \%$ \\
\hline 2022 & $11,36 \%$ & $5,88 \%$ & $\$ 27,65$ & $0,52 \%$ & $5,72 \%$ & $5,33 \%$ \\
\hline
\end{tabular}

Notas:

$\mathbf{E}\left(\boldsymbol{i}_{d}\right) \quad$ Tasa de interés doméstica

$\mathbf{E}\left(\boldsymbol{i}_{x}\right) \quad$ Tasa de interés externa

$\mathbf{F}_{t, n} \quad$ Tasa de cambio futura nominal

$\prod_{t, e} \quad$ Tasa de inflación externa proyectada

$\prod_{t, \boldsymbol{d}} \quad$ Tasa de inflación doméstica proyectada

$\boldsymbol{r}_{\boldsymbol{t}, \boldsymbol{r}} \quad$ Tasa real suponiendo equilibrio de Fisher (r)

Fuente: elaboración propia

c) Cálculo del CCPP nominal y real: uno de los insumos para estimar el CCPP es la tasa de rendimiento requerido por los propietarios o costo del capital propio. En este caso se utilizó el clásico modelo CAPM ${ }^{13}$. Para su construcción se consideró como proxy de tasa libre de riesgo $\left(r_{f}\right)$ el resultado de proyectar rendimientos de bonos soberanos domésticos en pesos (tabla 1). El rendimiento esperado de mercado $\left(E\left(r_{m}\right)\right)$ surge de considerar al promedio aritmético anualizado del índice bursátil MERVAL 25, serie 2007-2016 como proxy del rendimiento de la cartera de mercado. Finalmente, el coeficiente beta $\left(\beta_{i}\right)$ corresponde al publicado para empresas del sector por la consultora Economática ${ }^{\circledR}$. El rendimiento nominal en pesos requerido por los propietarios $\mathrm{E}\left(r_{i}\right)$ y el rendimiento nominal en dólares

13 El modelo presenta limitaciones, en particular para estimar tasas de rendimiento requerido en contextos emergentes. No es el objetivo del presente trabajo analizar las diferentes alternativas para la estimación de la tasa de descuento, entre otros ver Fama y French (2004) 
requerido por los propietarios $\mathrm{E}\left(r_{i, u \$}\right)$ (ecuación 44$)$ se presentan en la tabla 3. Con las series de inflación proyectada también se obtiene el rendimiento real de los propietarios $\mathrm{E}\left(r_{i, r}\right)$ (ecuaciones 7 y 8 ).

Tabla 2. Flujos de fondos proyectados nominales (moneda de cierre) y reales (iniciales) (valores en miles de pesos argentinos)

\begin{tabular}{|c|c|c|c|c|c|}
\hline $\begin{array}{l}\text { Flujo de fondos en moneda } \\
\text { corriente }\end{array}$ & Inicio & 1 & 2 & 3 & 4 \\
\hline Inversión fija & $\$-250.000,00$ & & & & \\
\hline EBITDA & & $\$ 206.823,70$ & $\$ 353.982,70$ & $\$ 846.572,27$ & $\$ 921.474,40$ \\
\hline Impuesto a las ganancias & & $\$-47.888,30$ & $\$-110.836,36$ & $\$-251.768,51$ & $\$-299.824,55$ \\
\hline Cambio CTO monetario & & $\$-54.728,08$ & $\$-101.520,84$ & $\$-59.588,67$ & $\$-124.442,01$ \\
\hline Cambio CTO no monetario & & $\$-254.552,25$ & $\$ 13.287,88$ & $\$ 89.526,30$ & $\$ 5.295,20$ \\
\hline $\begin{array}{l}\text { Flujo de fondos en mone- } \\
\text { da de cierre (ecuación 25) }\end{array}$ & $\$-250.000,00$ & $\$-150.344,92$ & $\$ 154.913,39$ & $\$ 624.741,39$ & $\$ 502.503,04$ \\
\hline $\begin{array}{l}\text { Flujo de fondos en moneda } \\
\text { inicial }\end{array}$ & Inicio & 1 & 2 & 3 & 4 \\
\hline $\begin{array}{l}\text { Flujo de fondos en mone- } \\
\text { da de cierre }\end{array}$ & $\$-250.000,00$ & $\$-150.344,92$ & $\$ 154.913,39$ & $\$ 624.741,39$ & $\$ 502.503,04$ \\
\hline $\begin{array}{l}\text { Coeficiente de inflación } \\
\text { (ecuación 6) }\end{array}$ & 1 & 1,193213673 & 1,361471913 & 1,511736189 & 1,645490008 \\
\hline $\begin{array}{l}\text { Flujo de fondos en mone- } \\
\text { da inicial (ecuación 26) }\end{array}$ & $\$-250.000,00$ & $\$-126.000,00$ & $\$ 113.783,76$ & $\$ 413.260,85$ & $\$ 305.382,01$ \\
\hline
\end{tabular}

Fuente: elaboración propia

Tabla 3. Estimación tasa de rendimiento requerida $\left(k_{e}\right)$

\begin{tabular}{|ccccccc|}
\hline$r_{\mathrm{f}}$ & $\mathrm{E}\left(r_{m}\right)$ & $\mathrm{E}\left(r_{m}\right)-r_{f}$ & $\beta_{i}$ & $\mathrm{E}\left(\mathrm{r}_{\mathrm{i}}\right)=\mathrm{k}_{\mathrm{e}}$ & $\mathrm{E}\left(\mathrm{r}_{\mathrm{i}, \mathrm{us}}\right)$ & $\mathrm{E}\left(\mathrm{r}_{\mathrm{i}, \mathrm{r}}\right)$ \\
\hline $22,83 \%$ & $41,68 \%$ & $18,85 \%$ & 0,7568 & $37,10 \%$ & $2,013 \%$ & $14,90 \%$ \\
$18,39 \%$ & $41,68 \%$ & $23,29 \%$ & 0,7568 & $36,02 \%$ & $2,409 \%$ & $19,21 \%$ \\
$15,80 \%$ & $41,68 \%$ & $25,88 \%$ & 0,7568 & $35,39 \%$ & $2,687 \%$ & $21,93 \%$ \\
$13,96 \%$ & $41,68 \%$ & $27,72 \%$ & 0,7568 & $34,94 \%$ & $2,893 \%$ & $23,97 \%$ \\
$12,53 \%$ & $41,68 \%$ & $29,15 \%$ & 0,7568 & $34,59 \%$ & $3,042 \%$ & $25,63 \%$ \\
$11,36 \%$ & $41,68 \%$ & $30,32 \%$ & 0,7568 & $34,31 \%$ & $3,130 \%$ & $27,04 \%$ \\
\hline
\end{tabular}

Fuente: elaboración propia

El aumento en la tasa real del costo del capital propio doméstico obedece al hecho de que el adicional por riesgo de mercado, expresado en moneda de 
cierre, aumenta a lo largo del tiempo manteniendo constante el coeficiente beta. No obstante, la inflación doméstica disminuye más que proporcionalmente en relación a la extranjera (tabla 1). Esto hace que el valor de $\mathrm{E}\left(r_{i, r}\right)$ aumente. Con los datos de la tabla 3 se calcula el CCPP nominal (ecuación 30), real directo (ecuación 31) e indirecto (ecuación 32) en pesos. En el caso bajo estudio, la tasa de la deuda nominal se supone equivalente al costo financiero para firmas de primera línea, a los efectos de tomar un valor cercano a la tasa libre de riesgo doméstico. Las tasas reales se obtienen a partir de las tasas nominales de la deuda, y el capital propio se obtiene luego de estimar las tasas nominales (ecuación 7). Se supone arbitrariamente una ratio de apalancamiento financiero con una relación de deuda (30\%) y capital (70\%). La tabla 4 presenta los resultados.

Tabla 4. Proyección del CCPP en términos nominales y reales

\begin{tabular}{|l|c|c|c|c|c|}
\hline Tasa de inflación proyectada & 1 & 2 & 3 & 4 & 5 \\
\hline Tasa de inflación & $19,32 \%$ & $14,10 \%$ & $11,04 \%$ & $8,85 \%$ & $7,14 \%$ \\
\hline Tasa de inflación acumulada & 1,1932 & 1,3614 & 1,5117 & 1,6454 & 1,7629 \\
\hline Tasas en términos reales & 1 & 2 & 3 & 4 & 5 \\
\hline $\mathrm{k}_{\mathrm{e}}$ (calculadas a partir de la nominal) & $14,90 \%$ & $19,21 \%$ & $21,93 \%$ & $23,97 \%$ & $25,63 \%$ \\
\hline $\mathrm{k}_{\mathrm{d}}$ (calculadas a partir de la nominal) & $11,00 \%$ & $10,00 \%$ & $10,00 \%$ & $9,00 \%$ & $9,00 \%$ \\
\hline $\mathrm{W}_{1}$ & 0,7 & 0,7 & 0,7 & 0,7 & 0,7 \\
\hline $\mathrm{W}_{2}$ & 0,3 & 0,3 & 0,3 & 0,3 & 0,3 \\
\hline CCPP nominal & $\mathbf{1}$ & $\mathbf{2}$ & $\mathbf{3}$ & $\mathbf{4}$ & $\mathbf{5}$ \\
\hline $\mathrm{k}_{\mathrm{e}}$ & $37,10 \%$ & $36,02 \%$ & $35,39 \%$ & $34,94 \%$ & $34,59 \%$ \\
\hline $\mathrm{k}_{\mathrm{d}}$ & $32,45 \%$ & $25,51 \%$ & $22,14 \%$ & $18,64 \%$ & $16,78 \%$ \\
\hline Tasa de impuesto & $35 \%$ & $35 \%$ & $35 \%$ & $35 \%$ & $35 \%$ \\
\hline ccpp nominal & $32,29 \%$ & $30,19 \%$ & $29,09 \%$ & $28,09 \%$ & $27,49 \%$ \\
\hline CCPP real & $\mathbf{1}$ & $\mathbf{2}$ & $\mathbf{3}$ & $\mathbf{4}$ & $\mathbf{5}$ \\
\hline ccpp real indirecto a partir nominal (ec.31) & $10,872 \%$ & $14,097 \%$ & $16,256 \%$ & $17,680 \%$ & $18,994 \%$ \\
\hline $\mathrm{k}_{\mathrm{e}}$ real & $14,90 \%$ & $19,21 \%$ & $21,93 \%$ & $23,97 \%$ & $25,63 \%$ \\
\hline $\mathrm{k}_{\mathrm{i}}$ real después de tax & $1,48 \%$ & $2,17 \%$ & $3,02 \%$ & $3,01 \%$ & $3,52 \%$ \\
\hline ccpp real método directo (ec.32) & $10,872 \%$ & $14,097 \%$ & $16,256 \%$ & $17,680 \%$ & $18,994 \%$ \\
\hline
\end{tabular}

Fuente: elaboración propia 
d) La determinación del valor de continuidad (valor terminal): en la tabla 6 se presentan las variables y resultados obtenidos correspondientes al valor de continuidad. Se supone que los flujos de fondos en T-1 se estabilizan en T (año 5). Los modelos usados son: (i) ZRG en moneda de cierre e inicial, respectivamente (A y B) (ec.39 y 40); con G=0 (ii) ZNPIM nominal con crecimiento equivalente (C) (ecuación 42) y crecimiento mayor (D), (ecuación 41) a la inflación. En ZNPI las inversiones incrementales generan un VAN $=0$.

El CCPP nominal y real en dólares (ecuación 44 y 48) se presenta en la tabla 5.

Tabla 5. Proyección del CCPP en dólares en términos nominales y reales

\begin{tabular}{|l|c|c|c|c|c|}
\hline CCPP en dólares & 1 & 2 & 3 & 4 & 5 \\
\hline Inflación proyectada EE. UU. & $1,05 \%$ & $0,94 \%$ & $0,84 \%$ & $0,73 \%$ & $0,63 \%$ \\
\hline A partir del ccpp nominal & $12,03 \%$ & $15,17 \%$ & $17,23 \%$ & $18,54 \%$ & $19,74 \%$ \\
\hline ccpp real en dólares (ec.44) & $10,87 \%$ & $14,10 \%$ & $16,26 \%$ & $17,68 \%$ & $18,99 \%$ \\
\hline
\end{tabular}

Fuente: elaboración propia

Tabla 6. Determinación del valor de continuidad ZRGM y ZNPI (valores en miles)

\begin{tabular}{|c|c|c|c|c|}
\hline Variables $\mathrm{T}(\mathrm{t}=5 \rightarrow \infty)$ & $\begin{array}{c}\text { A: ZRGM Flujo } \\
\text { MC }\end{array}$ & $\begin{array}{c}\text { B: ZRGM Flujo } \\
\text { MI }\end{array}$ & C: ZNPIM g $=\pi$ & $\begin{array}{c}\text { D: ZNPIM } \\
\text { con g }\end{array}$ \\
\hline ССРP real & $18,99 \%$ & $18,99 \%$ & $18,99 \%$ & $18,99 \%$ \\
\hline Inflación esperada & $7,14 \%$ & $7,14 \%$ & $7,14 \%$ & $7,14 \%$ \\
\hline CCPP nominal & & & $27,49 \%$ & $27,49 \%$ \\
\hline Tasa de crecimiento nominal & & & & $11,61 \%$ \\
\hline Tasa de reinversión & & & & $22,00 \%$ \\
\hline Flujos $t=4$. MI $t=4$ & & $\$ 305.382,01$ & & \\
\hline Flujos $t=5$, MI $t=5$ & $\$ 285.042,54$ & $\$ 285.042,54$ & & \\
\hline Flujos $t=4, M C t=4$ & $\$ 502.503,04$ & & $\$ 502.503,04$ & $\$ 502.503,04$ \\
\hline Flujos $t=5, \mathrm{MC} t=4$ & $\$ 502.503,04$ & & & \\
\hline Flujos $t=5, M C t=5$ & & & $\$ 538.359,60$ & $\$ 538.359,60$ \\
\hline VC valor nominal & $\ldots$ & - & $\$ 2.645 .564,75$ & $\$ 2.645 .564,75$ \\
\hline Efecto absorción & & & 1,7629 & 1,7629 \\
\hline Valor terminal MI & $\$ 1.500 .684,44$ & $\$ 1.500 .684,44$ & $\$ 1.500 .684,44$ & $\$ 1.500 .684,44$ \\
\hline
\end{tabular}

Notas: MC: moneda de cierre; MI: moneda de inicio

Fuente: elaboración propia 
Respecto de los flujos de fondos empleados, el supuesto general es que la magnitud proyectada para $t=4$ se mantiene constante en $t=5$. Por lo tanto, las correcciones monetarias son las siguientes:

I) ZRGM (MC) toma el flujo de fondos en $t=4$ en moneda de cierre de $\$ 502.503,04$. Se deflacta desde $t=5$ hasta $t=0$, por el coeficiente $1,76 \mathrm{y}$, por lo tanto, arroja un flujo de fondos expresado en moneda inicial de \$285.042,54,

II) El ZRGM (MI) trabaja con monedas en poder adquisitivo inicial. Así, los flujos de fondos nominales en $t=4 \$ 502.503,04$ son expresados en términos reales para el período $t=4$, siendo de $\$ 305.382,01$. Deflactados desde $t=5$ hasta $t=0$ ascienden a $\$ 285.042,54$,

III) ZNPIM con $g=\pi$ y $g \neq \pi$ toma el flujo de fondos nominal en $t=4$, de $\$ 502.503,04$. Se traslada la inflación proyectada para $t=5$, obteniendo un flujo de fondos esperados, expresado en moneda de dicho período de $\$ 538.359,60$.

Para todos los casos queda demostrado que los modelos son neutrales a la inflación, ya que arrojan el mismo valor.

e) El valor actual en moneda doméstica y extranjera, en términos nominales y reales: con la información de los cuadros precedentes, se proyectan las variables para estimar flujos nominales y reales expresados en dólares futuros, en términos nominales y reales. La tabla 1 provee los valores de: $i$ ) tasas de interés proyectadas local y extranjera; ii) inflación esperada local y extranjera; iii) tasa de cambio futura. Con las tablas 4 y 5 se obtiene el CCPP nominal para Argentina y Estados Unidos. La siguiente tabla presenta las cifras correspondientes las tasas de cambio nominal deflactado (ecuación 16) y real proyectado (ecuación 46).

Tabla 7. Tasa de cambio futura nominal, deflactada y real

\begin{tabular}{|l|c|c|c|c|c|c|}
\hline \multicolumn{1}{|c|}{ Proyecciones económicas } & base & 1 & 2 & 3 & 4 & 5 \\
\hline Var. nivel de precios Argentina (tabla 1) & & $19,3 \%$ & $14,1 \%$ & $11,0 \%$ & $8,8 \%$ & $7,1 \%$ \\
\hline Índice nivel general de precios Argentina & 1 & 1,19 & 1,36 & 1,51 & 1,65 & 1,76 \\
\hline Var. nivel de precios EE. UU. (tabla 1) & & $1,05 \%$ & $0,94 \%$ & $0,84 \%$ & $0,73 \%$ & $0,63 \%$ \\
\hline Índice nivel general de precios EE. UU. & 1 & 1,01 & 1,02 & 1,03 & 1,04 & 1,04 \\
\hline $\begin{array}{l}\text { Tasa de cambio futuro (en pesos de } \\
\text { cierre) }\end{array}$ & $\$ 15,50$ & $\$ 18,30$ & $\$ 20,69$ & $\$ 22,78$ & $\$ 24,62$ & $\$ 26,21$ \\
\hline
\end{tabular}


Descuento de flujo de fondos e inflación para la valoración de empresas en dos monedas

\begin{tabular}{|l|c|c|c|c|c|c|}
\hline \multicolumn{1}{|c|}{ Proyecciones económicas } & base & 1 & 2 & 3 & 4 & 5 \\
\hline Tasa de cambio futuro (deflactado) & & $\$ 15,34$ & $\$ 15,20$ & $\$ 15,07$ & $\$ 14,96$ & $\$ 14,87$ \\
\hline $\begin{array}{l}\text { Tasa de cambio futuro (en pesos ini- } \\
\text { ciales) }\end{array}$ & $\$ 15,50$ & $\$ 15,50$ & $\$ 15,50$ & $\$ 15,50$ & $\$ 15,50$ & $\$ 15,50$ \\
\hline $\begin{array}{l}\text { Tasa interés proyectada Argentina } \\
\text { (tabla 1) }\end{array}$ & $22,8 \%$ & $18,4 \%$ & $15,8 \%$ & $14,0 \%$ & $12,5 \%$ \\
\hline $\begin{array}{l}\text { Tasa interés proyectada EE. UU. } \\
\text { (tabla 1) }\end{array}$ & $4,0 \%$ & $4,7 \%$ & $5,2 \%$ & $5,5 \%$ & $5,7 \%$ \\
\hline CPPC nominal Argentina (tabla 4) & & $32,3 \%$ & $30,2 \%$ & $29,1 \%$ & $28,1 \%$ & $27,5 \%$ \\
\hline CPPC nominal en EE. UU. (tabla 5) & & $12,0 \%$ & $15,2 \%$ & $17,2 \%$ & $18,5 \%$ & $19,7 \%$ \\
\hline
\end{tabular}

Fuente: elaboración propia

En la siguiente tabla se presenta los resultados obtenidos con la aplicación del modelo sobre flujos expresados en moneda de cierre e inicial.

Tabla 8. Valor actual flujos de fondos nominal y real en moneda doméstica

\begin{tabular}{|l|c|c|c|c|c|c|}
\hline \multicolumn{1}{|c|}{ Flujo de fondos } & \multicolumn{1}{c|}{ base } & 1 & 2 & 3 & 4 & 5 \\
\hline $\begin{array}{l}\text { En moneda de cierre } \\
\text { (Tabla 2 y 6) }\end{array}$ & $\$-250.000,0$ & $\$-150.344,9$ & $\$ 154.913,4$ & $\$ 624.741,4$ & $\$ 502.503,0$ & $\$ 2.645 .564,7$ \\
\hline ccpp nominal & & $32,3 \%$ & $30,2 \%$ & $29,1 \%$ & $28,1 \%$ & $27,5 \%$ \\
\hline $\begin{array}{l}\text { Valor actual flujo no- } \\
\text { minal }\end{array}$ & $\$-250.000,0$ & $\$-113.644,5$ & $\$ 89.946,2$ & $\$ 281.002,4$ & $\$ 176.451,8$ & $\$ 728.695,8$ \\
\hline Valor actual neto & $\mathbf{\$ 9 1 2 . 4 5 1 , 6}$ & & & & & \\
\hline En moneda inicial & $\$-250.000,0$ & $\$-126.000,0$ & $\$ 113.783,8$ & $\$ 413.260,9$ & $\$ 305.382,0$ & $\$ 1.500 .684,4$ \\
\hline ccpp real & & $10,9 \%$ & $14,1 \%$ & $16,3 \%$ & $17,7 \%$ & $19,0 \%$ \\
\hline Valor actual flujo real & $\$-250.000,0$ & $\$-113.644,5$ & $\$ 89.946,2$ & $\$ 281.002,4$ & $\$ 176.451,8$ & $\$ 728.695,8$ \\
\hline Valor actual neto & $\mathbf{\$ 9 1 2 . 4 5 1 , 6}$ & & & & & \\
\hline
\end{tabular}

Fuente: elaboración propia

A continuación, y utilizando los valores expuestos en la tabla 7 , se valúa en moneda extranjera. Para ello, los flujos domésticos expresados en moneda de cierre, son convertidos a dólares con la ecuación 43. El CCPP se estima aplicando la ecuación 44, y el valor actual en dólares, utilizando la ecuación 45. En relación con magnitudes reales, los flujos domésticos en moneda inicial se convierten a dólar futuro real, aplicando la ecuación 47. El CCPP extranjero en moneda inicial y el valor actual son calculados utilizando las ecuaciones 48 y 49 . 
Tabla 9. Valor actual flujo de fondos nominales y reales en moneda extranjera

\begin{tabular}{|c|c|c|c|c|c|c|}
\hline $\begin{array}{l}\text { Flujo de fondos en moneda } \\
\text { doméstica }\end{array}$ & base & 1 & 2 & 3 & 4 & 5 \\
\hline $\begin{array}{l}\text { En moneda de cierre } \\
\text { (tabla } 2 \text { y 6) }\end{array}$ & $\$-250.000,0$ & $\$-150.344,9$ & $\$ 154.913,4$ & $\$ 624.741,4$ & $\$ 502.503,0$ & $\$ 2.645 .564,7$ \\
\hline $\begin{array}{l}\text { En moneda inicial } \\
\text { (Tabla } 2 \text { y 6) }\end{array}$ & $\$-250.000,0$ & $\$-126.000,0$ & $\$ 113.783,8$ & $\$ 413.260,9$ & $\$ 305.382,0$ & $\$ 1.500 .684,4$ \\
\hline $\begin{array}{l}\text { Flujo de fondos en } \\
\text { moneda extranjera }\end{array}$ & base & 1 & 2 & 3 & 4 & 5 \\
\hline En divisa de cierre & $\$-16.129,0$ & $\$-8.214,3$ & $\$ 7.487,8$ & $\$ 27.423,6$ & $\$ 20.413,4$ & $\$ 100.943,2$ \\
\hline ссpp en dólares nominal & & $12,03 \%$ & $15,17 \%$ & $17,23 \%$ & $18,54 \%$ & $19,74 \%$ \\
\hline $\begin{array}{l}\text { Valor actual flujo } \\
\text { nominal }\end{array}$ & $\$-16.129,0$ & $\$-7.331,9$ & $\$ 5.803,0$ & $\$ 18.129,2$ & $\$ 11.384,0$ & $\$ 47.012,6$ \\
\hline $\begin{array}{l}\text { Valor actual neto en } \\
\text { dólares }\end{array}$ & $\$ 58.867,84$ & & & & & \\
\hline En divisa inicial & $\$-16.129,0$ & $\$-8.129,0$ & $\$ 7.340,9$ & $\$ 26.662,0$ & $\$ 19.702,1$ & $\$ 96.818,4$ \\
\hline cсpp en dólares real & & $10,9 \%$ & $14,1 \%$ & $16,3 \%$ & $17,7 \%$ & $19,0 \%$ \\
\hline Valor actual flujo real & $\$-16.129,0$ & $\$-7.331,9$ & $\$ 5.803,0$ & $\$ 18.129,2$ & $\$ 11.384,0$ & $\$ 47.012,6$ \\
\hline $\begin{array}{l}\text { Valor actual neto en } \\
\text { dólares }\end{array}$ & $\$ 58.867,84$ & & & & & \\
\hline
\end{tabular}

Fuente: elaboración propia

La siguiente tabla expone los resultados correspondientes a cocientes entre magnitudes expresadas en diferentes monedas, pero con el mismo poder adquisitivo y pertinencia temporal. Los resultados obtenidos son: a) tasa de cambio futura para magnitudes futuras expresadas en moneda de cierre, b) tasa de cambio spot para valores para magnitudes futuras expresadas en moneda de inicio, c) tasa spot para el cociente de valores actuales en diferentes monedas (ecuaciones 50 y 51)

Tabla 10. Cociente de magnitudes expresadas en dos monedas, en términos nominales y reales

\begin{tabular}{|lcccccc|}
\hline Magnitudes & $\mathbf{0}$ & $\mathbf{1}$ & $\mathbf{2}$ & $\mathbf{3}$ & $\mathbf{4}$ & $\mathbf{5}$ \\
FFL(\$)/FFL(uS) cierre & $\$ 15,50$ & $\$ 18,30$ & $\$ 20,69$ & $\$ 22,78$ & $\$ 24,62$ & $\$ 26,21$ \\
FFL(\$)/FFL(uS)inicia & $\$ 15,50$ & $\$ 15,50$ & $\$ 15,50$ & $\$ 15,50$ & $\$ 15,50$ & $\$ 15,50$ \\
VA(\$)/VA(u\$) & $\$ \mathbf{1 5 , 5 0}$ & & & & & \\
\hline
\end{tabular}

Fuente: elaboración propia 


\section{CONCLUSIONES}

Tomando como pilares las teorías de paridad, se desarrolló un marco conceptual consistente aplicando el descuento de flujos de fondos en dos monedas. El modelo compila y entrelaza todos los aspectos técnicos relativos al tratamiento de la inflación y expresión de cifras en términos nominales y reales sobre magnitudes financieras, costo del capital y valor terminal. Mediante un estudio de casos se ilustra su respectivo funcionamiento y consistencia, prestando especial atención al tratamiento de las inversiones incrementales, ahorros fiscales e inflación, correcta especificación de la tasa de crecimiento para estimar valores terminales, paridades cambiarias y consistencia de valores actuales. La lógica inherente del modelo y sus soluciones técnicas se encuentran atravesadas por las ecuaciones de paridad; por lo tanto, los resultados obtenidos corroboran la consistencia del marco conceptual propuesto ya que:

a) Al trabajar en una única moneda se obtiene el mismo valor actual, independientemente que las magnitudes se expresen en términos nominales o reales, producto de respetar las teorías de paridad, las relaciones de tasas y la consistencia de la técnica de descuento de flujos de fondos en moneda inicial y de cierre.

b) La valoración con dos monedas, dado que las diferencias entre flujos de fondos y valores actuales es explicada por la variable tasa de cambio. Este es el nexo entre tasas e inflación esperada entre el mercado local y extranjero. El modelo propuesto presenta consistencia. Para valores en moneda inicial, la tasa de cambio spot explica las diferencias; para valores en moneda de cierre es la tasa de cambio futuro.

En este trabajo se utilizó el estudio de casos como metodología para la obtención de resultados que corroboren las ecuaciones presentadas en la sección 1. No obstante, existen ciertas limitaciones en los resultados obtenidos, ya que, si bien conceptualmente el modelo expresa un equilibrio de largo plazo, a través de relaciones de paridad, empíricamente debe contrastarse el cumplimiento de las relaciones manifiestas. Estas relaciones se ajustan en el caso de activos financieros; no obstante, en futuras líneas de investigación, se puede analizar la eficacia de las mismas para valorar empresas en marcha.

\section{BIBLIOGRAFÍA}

Argandoña, A. (2013). Irvin Fisher: un gran economista. En: Working Paper WP-1082, IESE insight 11/2013, $44 \mathrm{p}$.

Booth, L. (2007). Capital Cash Flow, APV and Valuation. En: European Financial Management, Vol. 13, No. 1, p. 29-48. 
Bradley, M. y Gregg, J. (2008). Expected Inflation and the Constant Growth Valuation Model. En: Journal of Applied Corporate Finance, Vol. 20, No. 2, p. 66-78.

Brealey, R. Myers,S. y Allen, F. (2006). Principles of Corporate Finance McGraw Hill Custom Publishing, 8a ed., 896p.

Castro Monge, E. (2010). El estudio de casos como metodología de investigación y su importancia en la dirección y administración de empresas. En: Revista Nacional de Administración, Vol. 2, No. 1, p. 31-54.

Copeland, T.; Koller, T. y Murrin, J. (2000). Valuation: Measuring and Managing the Value of Companies. New York: Wiley, 3a ed., 508p.

Damodaran, A. (2006). Damodaran on Valuation. New York, United State: John Wiley E Sons, 2a ed., 696p.

Emery, D. y Finnerty, J. (2007). Corporate Financial Management. New Jersey; Prentice Hall, $3 a$ ed., $825 p$.

Esandi, J.; Milanesi, G. y Pesce, G. (2012). Análisis de escenario y opciones reales: un caso de aplicación para empresas de base tecnológica. XXXII Jornadas Sociedad Argentina de Docentes en Administración Financiera SADAF, p. 63-82.

Fabozzi, F. y Fabozzi, D. (1996). Bond Markets, Analysis and Strategies. New Jersey, Prentice Hall, 303p.

Fama, E. y French, K. (2004). The capital asset pricing model: Theory and evidence. En: Journal of Economics Perspectives, Vol. 18, No. 3, p.25-46.

Fernández, P. (2015). Valoración de Empresas y Sensatez. IESE Business School, Universidad de Navarra, 5 a ed.

Fisher, I. (1965). The Theory of Interest. New York; NY: August Kelley, 183p.

Gordon, M. (1962). The Investment, Financing and Valuation of the Corporation. Illinois: Irwin Homewood, 252p.

Hull, J. (2005). Futures, Options and other Derivatives. New Jersey: Prentice Hall, 5a ed., 756p.

López Dumrauf, G. (2014a). Análisis cuantitativo de bonos. Buenos Aires: Alfaomega, 442p.

López Dumrauf, G. (2014b). Currency Choice in Valuation: an Approach for Emerging Markets. En: The Business and Economics Research Journal, Vol. 7, No.1, p. 11-22.

Miller, K. y Waller, G. (2003). Scenarios, Real Options and Integrated Risk Management. En: Journal of Long Range Planning, Vol. 36, No.1, p. 93-107.

Modigliani, F. y Cohn, R. (1984). Inflation and Corporate Financial Management. En: MIT Sloan School Working Paper, 37 p.

Pratt, S. y Grabowski, R. (2008). Cost of Capital: Applications and Examples. New Jersey, John Wiley $\&$ Sons, 3a ed., 448p. 
Descuento de flujo de fondos e inflación para la valoración de empresas en dos monedas

Rappaport, A. (1998). Creating Shareholder Value. New York, The Free Press, 2a ed., 224p.

Ruback, R. (2002). Capital Cash Flow: A simple approach to valuing risky cash flow. En: Financial Management, Vol. 31, No. 2, p. 85-103.

Than, J. y Vélez-Pareja, I. (2011). Will the deflated WACC please stand up? And the real WACC should sit down. En: Cuadernos Latinoamericanos de Administración, Vol. 6, No. 12, julio-diciembre, p. $\overline{1-17}$.

Titman, S. y Grinblatt, M. (2002). Financial Markets and Corporate Strategy. NY: McGraw-Hill, 892p.

Vélez-Pareja, I. (2006). Valoración de flujos de caja en inflación. El caso de la regulación en el Banco Mundial. Academia. En: Revista Latinoamericana de Administración, No. 36, p. 24-49.

Wright, G. Cairns, G. y Goodwin, P. (2009). Teaching scenario planning: Lessons from practice in academe and business. En: European Journal of Operational Research, Vol. 194, No.1, p. 323-335. 


\section{ANEXOS}

Tabla A1. TIR y duración modificada. Bonos soberanos nominados en pesos

\begin{tabular}{|l|l|l|l|}
\hline Bonos Nominados en Pesos (30/9/2016) & Sticker & TIR & DM \\
\hline Bogar 2018+CER & NF18 & $4,07 \%$ & 0,7 \\
\hline Bonos de consolidacion 2\%+CER & PR13 & $2,94 \%$ & 3,47 \\
\hline BONCER 2021+CER & TC21 & $2,63 \%$ & 4,41 \\
\hline Discount \$ Ley Arg reestructuración 2005 & DICP & $3,90 \%$ & 8,91 \\
\hline PAR \$ Ley Arg reestructuración 2005 & PARP & $5,01 \%$ & 14,02 \\
\hline Cuasipar \$ Ley Arg reestructuración 2005 & CUAP & $4,22 \%$ & 15,55 \\
\hline
\end{tabular}

Fuente: Instituto Argentino de Mercados de Capitales, 30/9/2016

Tabla A2. TIR y duración modificada. Bonos soberanos nominados en dólares

\begin{tabular}{|l|c|c|c|}
\hline Bonos Nominados en Dólares (30/9/2016) & Sticker & TIR & DM \\
\hline Bonar 2016 6\% & AD16 & $2,14 \%$ & 0,15 \\
\hline Bonar X & AA17 & $3,94 \%$ & 0,5 \\
\hline Global 2017 ley U\$ & GI17 & $3,15 \%$ & 0,62 \\
\hline Bonar 2020 & AO20 & $4,36 \%$ & 3,31 \\
\hline Bonos cancelación deuda 6,85\% 31/3/2016 & AA21 & $5,11 \%$ & 3,74 \\
\hline Bonar 2024 & AY24 & $5,24 \%$ & 3,98 \\
\hline Bonos cancelación deuda 7,50\% 31/3/2016 & AA26 & $5,94 \%$ & 6,64 \\
\hline Discount u\$ Ley NY reestructuración 2005 & DICY & $6,60 \%$ & 7,73 \\
\hline Discount u\$ Ley Arg reestructuración 2005 & DICA & $6,61 \%$ & 7,74 \\
\hline PAR u\$ Ley Arg reestructuración 2005 & PARA & $6,70 \%$ & 11,91 \\
\hline PAR u\$ Ley NY reestructuración 2005 & PARY & $6,54 \%$ & 11,97 \\
\hline Bonos cancelación deuda 7,62\% 31/3/2016 & AA46 & $6,75 \%$ & 12,03 \\
\hline
\end{tabular}

Fuente: Instituto Argentino de Mercado de Capitales, 30/9/2016 
Descuento de flujo de fondos e inflación para la valoración de empresas en dos monedas

Tabla A3. Proyección de ventas-producción, precios, costos unitarios, ingresos totales, costos totales y existencias en moneda de cierre

\begin{tabular}{|c|c|c|c|c|c|}
\hline Proyección Magnitudes & base & 1 & 2 & 3 & 4 \\
\hline Producción (unidades) & 600 & 600 & 600 & 800 & 800 \\
\hline Ventas (unidades) & & 400 & 600 & 800 & 800 \\
\hline Coeficientes nivel de inflación & & 1,1932 & 1,3614 & 1,5117 & 1,6454 \\
\hline Precio de venta & $\$ 1.500,00$ & $\$ 1.789,82$ & $\$ 2.042,21$ & $\$ 2.267,60$ & $\$ 2.468,24$ \\
\hline Variación & & $19,32 \%$ & $14,10 \%$ & $11,04 \%$ & $8,85 \%$ \\
\hline Costo variable & $\$ 500,00$ & $\$ 596,61$ & $\$ 680,74$ & $\$ 755,87$ & $\$ 822,75$ \\
\hline Costo fijo (miles) & $\$ 340.000,00$ & $\$ 405.692,65$ & $\$ 462.900,45$ & $\$ 513.990,30$ & $\$ 559.466,60$ \\
\hline Costo total (miles) & $\$ 640.000,00$ & $\$ 763.656,75$ & $\$ 871.342,02$ & $\$ 967.511,16$ & $\begin{array}{c}\$ \\
1.053 .113,60\end{array}$ \\
\hline Costo total unitario & $\$ 1.066,67$ & $\$ 1.272,76$ & $\$ 1.452,24$ & $\$ 1.209,39$ & $\$ 1.316,39$ \\
\hline Bienes de cambio final (unidades) & 0 & 200 & 200 & 200 & 200 \\
\hline Bienes de cambio EI & & \$- & $\$ 254.552,25$ & \$ $290.447,34$ & $\$ 241.877,79$ \\
\hline Bienes de cambio EF & & $\$ 254.552,25$ & $\$ 290.447,34$ & \$ 241.877,79 & $\$ 263.278,40$ \\
\hline Costo total erogable & & $\$ 763.656,75$ & $\$ 871.342,02$ & $\$ 967.511,16$ & $\begin{array}{c}\$ \\
1.053 .113,60\end{array}$ \\
\hline $\begin{array}{l}\text { Costo productos vendidos (valores } \\
\text { históricos) }\end{array}$ & & $\$ 509.104,50$ & $\$ 835.446,93$ & $\begin{array}{c}\$ \\
1.016 .080,71\end{array}$ & \$ $1.031 .712,99$ \\
\hline $\begin{array}{l}\text { Costo productos vendidos (valores } \\
\text { corrientes) }\end{array}$ & & $\$ 509.104,50$ & $\$ 871.342,02$ & $\$ 967.511,16$ & $\begin{array}{c}\$ \\
1.053 .113,60\end{array}$ \\
\hline Tasa de inflación & & $19 \%$ & $14 \%$ & $11 \%$ & $9 \%$ \\
\hline $\begin{array}{l}\text { Indice de precio mayorista (acu- } \\
\text { mulada) }\end{array}$ & 1 & 1,1932 & 1,3614 & 1,5117 & 1,6454 \\
\hline $\begin{array}{l}\text { Indice de precios de venta (acu- } \\
\text { mulada) }\end{array}$ & 1 & 1,1932 & 1,3614 & 1,5117 & 1,6454 \\
\hline $\begin{array}{l}\text { Índice de precios de costo } \\
\text { (acumulada) }\end{array}$ & 1 & 1,1932 & 1,3614 & 1,5117 & 1,6454 \\
\hline
\end{tabular}

Fuente: elaboración propia 
Tabla A4. Proyección resultados operativos, determinación del impuesto a las ganancias y capital de trabajo

\begin{tabular}{|c|c|c|c|c|}
\hline Resultados Operativo Proyectado & 1 & 2 & 3 & 4 \\
\hline Ventas & $\$ 715.928,20$ & $\$ 1.225 .324,72$ & $\$ 1.814 .083,43$ & $\$ 1.974 .588,01$ \\
\hline $\begin{array}{l}\text { Costo productos vendidos (valores } \\
\text { corrientes) }\end{array}$ & $\$ 509.104,50$ & $\$ 871.342,02$ & $\$ 967.511,16$ & $\$ 1.053 .113,60$ \\
\hline EBITDA & \$ 206.823,70 & $\$ 353.982,70$ & $\$ 846.572,27$ & $\$ 921.474,40$ \\
\hline Depreciaciones & $\$ 83.524,96$ & $\$ 99.663,12$ & $\$ 118.919,40$ & $\$ 141.896,25$ \\
\hline Impuesto a las ganancias & $\$ 47.888,30$ & $\$ 110.836,36$ & $\$ 251.768,51$ & $\$ 299.824,55$ \\
\hline Ganancias operativas proyectadas & $\$ 75.410,45$ & $\$ 143.483,22$ & $\$ 475.884,36$ & $\$ 479.753,60$ \\
\hline Determinación Impuesto a las ganancias & 1 & 2 & 3 & 4 \\
\hline Ventas & $\$ 715.928,20$ & \$1.225.324,72 & $\$ 1.814 .083,43$ & $\$ 1.974 .588,01$ \\
\hline $\begin{array}{l}\text { Costo productos vendidos (valores } \\
\text { históricos) }\end{array}$ & $\$ 509.104,50$ & $\$ 835.446,93$ & $\$ 1.016 .080,71$ & $\$ 1.031 .712,99$ \\
\hline Depreciaciones & $\$ 70.000,00$ & $\$ 73.202,48$ & $\$ 78.664,12$ & $\$ 86.233,43$ \\
\hline Ganancia gravable & $\$ 136.823,70$ & $\$ 316.675,31$ & $\$ 719.338,59$ & $\$ 856.641,58$ \\
\hline $\begin{array}{l}\text { Impuesto a las ganancias del } \\
\text { período }\end{array}$ & $\$ 47.888,30$ & $\$ 110.836,36$ & $\$ 251.768,51$ & $\$ 299.824,55$ \\
\hline $\begin{array}{l}\text { Impuesto a pagar al final de cada } \\
\text { período }\end{array}$ & $\$ 47.888,30$ & $\$ 62.948,06$ & $\$ 140.932,15$ & $\$ 48.056,05$ \\
\hline Capital de trabajo operativo & 1 & 2 & 3 & 4 \\
\hline Créditos por ventas & $\$ 178.982,05$ & $\$ 306.331,18$ & $\$ 453.520,86$ & $\$ 493.647,00$ \\
\hline Bienes de cambio final & $\$ 254.552,25$ & $\$ 290.447,34$ & $\$ 241.877,79$ & $\$ 263.278,40$ \\
\hline Bienes de cambio inicial reexpresado & \$ - & $\$ 303.735,23$ & $\$ 331.404,09$ & $\$ 268.573,60$ \\
\hline Deudas comerciales & $\$ 76.365,68$ & $\$ 87.134,20$ & $\$ 96.751,12$ & $\$ 105.311,36$ \\
\hline Impuestos a pagar & $\$ 47.888,30$ & $\$ 62.948,06$ & $\$ 140.932,15$ & $\$ 48.056,05$ \\
\hline Capital de trabajo operativo & $\$ 309.280,33$ & $\$ 446.696,26$ & $\$ 457.715,38$ & $\$ 603.558,00$ \\
\hline CTO monetario & $\$ 54.728,08$ & $\$ 156.248,92$ & $\$ 215.837,59$ & $\$ 340.279,60$ \\
\hline Cambio CTO monetario & $\$ 54.728,08$ & $\$ 101.520,84$ & $\$ 59.588,67$ & $\$ 124.442,01$ \\
\hline CTO no monetario & $\$ 254.552,25$ & $\$ 290.447,34$ & $\$ 241.877,79$ & $\$ 263.278,40$ \\
\hline Cambio CTO no monetario & $\$ 254.552,25$ & $\$-13.287,88$ & $\$-89.526,30$ & $\$-5.295,20$ \\
\hline
\end{tabular}

Fuente: elaboración propia 